\title{
Herbal Immersion Oil for Microscopic Identification of Malaria Parasites
}

\author{
Ayushi Rastogi ${ }^{1,3}$, V.K. Dua ${ }^{1}$, V.K. Varshney $^{2}$, N.C. Gupta ${ }^{1}$ and Sumit Kumar ${ }^{3}$ \\ ${ }^{1}$ National Institute of Malaria Research, Field Station, BHEL, Haridwar, India \\ ${ }^{2}$ Chemistry Department, Forest Research Institute, Dehradun, India \\ ${ }^{3}$ Shri Venketeshwara University, Gajraula, Amroha, India \\ *Corresponding author
}

\section{A B S T R A C T}

Oils from thirty-two plants were extracted using solvent extraction/steam distillation methods. All oils along with 11 blended oil mixtures were tested for their immersion oil

Keywords

Malaria

parasites,

Immersion oil,

Plant oil

mixture.

Article Info

Accepted:

21 June 2017

Available Online:

10 August 2017 properties for the detection of malaria parasites. The physicochemical properties required for an immersion oil including density, viscosity, refractive index and acid value of five oils namely as Ocimum basilicum, Pogostemon cablin, Papaver somniferum Ricinus communis and Valeriana jatamansi and blended mixture 6 were determined. Stability of above stated plant oils and blended mixture 6 , with time showed that all were stable under the observation period while non-dryness of these oils and blended mixture 6 , represented very good criteria for the non-drying less than $1 \%$ variation in weight. GC-MS analysis of mixture 6 clearly identified compounds namely alpha copane, trans-Caryophyllene Linalool L, Estragole, hexadecanoic acid phenyl methyl ester and 9, 12-Octadecadienoic acid and besides other minor components peaks. No peak of castor oil was detected due to lack of sample character and detection. Summary of in-house validation of blended mixture 6 classified as very good immersion oil (range 67-81\%) and good (19-33\%) for the examination of malaria parasites while the results of external validation clearly revealed that the developed plant oil mixture 6 possessed very good property as immersion oil for the examination of malaria parasites.

\section{Introduction}

Conventional immersion oils typically contain polychlorinated biphenyls (PCBs) which when blended with mineral oil and viscosity adjusting compounds provide generally useful immersion oil having many of the ideal characteristic. In recent years, however, PCB's have been discovered to be carcinogenic, a hazard to the human environment, and are generally regarded as toxic. Furthermore, PCB's are difficult to dispose off after use since they are extremely stable and non -biodegradable (Liva, 1986).
Attempts were also made to use oils from natural resources like plants. Cedar wood oil having a refractive index of 1.495 to 1.510 (British Pharmacopeia, 1963) was widely used for many years as immersion oil. Cedarwood oil was a mixture of organic compounds considered generally safe by the FDA as a food additive preservative and also used as an anti-bacterial, fungicide. However, studies have shown that prolonged exposure to high levels of cedar-wood oil can cause liver and pulmonary toxicity (FAO, 1995). In addition 
to this the cost wise cedar wood oil was expensive. In India, Victor et al., (2005) made an attempt to identify cost effective, but qualitative immersion oil for microscopy and photo microscopy. Pure castor oil and refined sunflower oil have been tried along with commercially available immersion oil on GTG banded chromosome preparations. Application of castor oil has been recommended as alternative to synthetic immersion oil. A review of the literature revealed that a limited work has been carried out to explore the possibility of using plant oils as an immersion oil for microscopy in spite of the fact that India has huge floura and fauna of plants. Attempts were made to screen different plants oils and combination of plant oils for their immersion oil property for microscopic identification of malaria parasites.

\section{Materials and Methods}

Thirty-two plants based on the physico chemical properties reported in the literature were collected from various parts of India in consultation with Forest Research Institute, Dehradun. Scientific name, family and common names, raw material used, extraction method of the plants under investigation are given in table 1. Plants were dried in shades and oils were extracted from the plants using solvent extraction (Soxhlet Extraction) and steam distillation methods.

\section{Microscopic examination of blood slides using plant oils as immersion oil}

All oils were examined for their immersion oil properties for the microscopic identification of malaria parasites. Stained blood slides were examined under microscope at $100 \mathrm{x}$ objective lens for detecting the malaria parasites. Different malaria parasite namely as Plasmodium falciparum/ Plasmodium vivax stages including gametocyte, rings, and schizont were examined by using immersion oil/plant oils.

Although microscopic examination is a manual qualitative approach, attempts were made to grade all plant oils for their performance based on clarity, sharpness and contrast of parasite stages. Based on microscopic examination of malaria parasite, only five plants oils showed good results as immersion oil. Eleven combinations of above five plants oils with different proportions were also prepared and microscopic tested of malaria parasites. The different compositions of five oils were as follow:

Mixture 1 Consisted of fifty percent each of Ocimum basilicum and Papaver somniferum (50:50), Mixture 2: Composed of fifty percent each of Pogostemon cablin and Papaver somniferum (50:50), Mixture 3: 25\% Ocimum basilicum and 25\% Pogostemon cablin oil and 50\% oil Papaver somniferum (25:25:50), Mixture 4: Ocimum basilicum oil was added into Ricinus communis oil at the ratio of 50:50, Mixture 5: The mixture was made from fifty percent of Pogostemon cablin and Ricinus communis oil (50:50), Mixture 6: It consisted of twenty five percent of each four of Pogostemon cablin, Ocimum basilicum, Papaver somniferum and Ricinus communis (25:25:25:25), Mixture 7: Papaver somniferum was blended with Ricinus communis (50:50), Mixture 8: It was a combination of three different oils namely as Ocimum basilicum, Pogostemon cablin and Ricinus communis at the ratio of $33: 33: 34$, Mixture 9: The thirty-five percent of Ocimum basilicum, Pogostemon cablin was blended with thirty percent of Ricinus communis., Mixture 10: It is composed of twenty-five percent of each of four different oils namely as Ocimum basilicum, Pogostemon cablin, Ricinus communis and Valeriana jatamansi and Mixture 11. Thirty-four percent of Valeriana jatamansi oil was mixed with 
thirty-three percent of Papaver somniferum and Ricinus communis oil.

\section{Determination of physico-chemical properties of different oils}

\section{Density}

The density of the microscopically selected plant oils/blended mixtures was measured by specific gravity bottles (relative density bottles) method. Experiments were conducted at room temperature.

\section{Acid value}

Acid value of different oils was determined as reported earlier (Barkatullah et al., 2012).

\section{Viscosity}

The Viscosity of the microscopically selected plant oils/blended mixtures were measured at the shear rate by using a D.V-iii ultraprogrammable rheometer (Brookfield Engineering Labs, U.S.A).The viscosity was determined by using different spindle no.18, 21 and 27 and different shear rates, ranging from 1.7-4.65 $\mathrm{s}^{-1}$. Nzikou et al., (2007) method was used to measure the different oil viscosities.

\section{Refractive index}

The refractive index of microscopically selected immersion oils/blended mixtures oils were determined by using refractometer based on the principle of the critical angle using diffused daylight.

\section{Non-dryness}

The non-dryness was determined by performing a test at $30 \mathrm{C}$ for 24 hours in accordance with JIS C 2201 Test of evaporation amount of "electrical insulating oils" (Fujioka et al., 2009) and was evaluated based on the following two levels. Good (0): evaporation amount of less than $1 \%$ by mass Poor (x): evaporation amount of $1 \%$ by mass or more.

\section{Stability}

Microscopically selected plant oils/ blended mixtures were kept at 25 and $37^{\circ} \mathrm{C}$ to investigate the stability of oils as an immersion oil. All the five plant oils/ blended mixtures were tested for the microscopic examination of malaria parasite at the intervals of five weeks.

\section{Gas chromatography- mass spectrometry (GCMS) analysis of plant oils blended mixtures}

GC-MS analysis for the separation and identification of plant oils /blended oils was performed at Department of Chemistry, Forest Research Institute, Dehradun. The sample was analyzed using a Shimadzu GC2010 gas chromatograph coupled to a QP 2010 mass-selective detector with capillary column BP-20 $(30 \mathrm{~m}$ in length, $0.25 \mathrm{~mm}$ i.d, and $0.25 \mu \mathrm{m}$ in thickness). GCMS conditions were used as described by Dua et al., (2013). Different peaks of gas chromatographic analysis were identified using NIST, WELY and SZTERP software library of mass spectra.

\section{Validation}

\section{In -house validation}

Four microscopist from National Institute of Malaria Research, field unit, BHEL, Haridwar were selected to examine the blood slides for malaria parasite using mixture 6. Each microscopist was provided 10 malaria positive blood slides with Plasmodium falciparum infection having ring/gametocyte and 10 Plasmodium vivax infections with ring, gametocytes and trophozoite stages. 


\section{External validation}

Each participating institutes was provided mixture 6 as immersion oil for the evaluation report. It is suggested to examine minimum of ten blood slide each with Plasmodium falciparum and Plasmodium vivax infection having different stages of parasites for evaluation of particular oil.

\section{Results and Discussion}

The maximum $\%$ yield was obtained from Junglans regia, (51), followed by Papaver somniferum (45),Michalia champaca (37), Terminellia belerica (33), Aleurites fordii (32) Sapindus mukrossi (31), Pongammia pinnata (31), Moringa oleferia (29), Ceiba pentandra (28), Sterculia foetida (28), Jatropa curcas (28), Ricinus communis (25), Terminelia chebulla (22), Bauhinia purpurea (16), Mimusops elengi (15), Bauhinia retusa (15), Pinus roxberghii (15), Pithecolobium dulci (14), Olea europaea (14), Psorylia coryfolia (8). Cesalpinia boundecella (2), Cymbopogon martini (1.2), Cedrus deodara (1.2), Melaleuca alternifolia (1.1), Cymbopogon citratus (1.1), Eucalyptus citriodora (0.9), Valeriana jatamansi (0.87), Cymbopogon nardus (0.8), Pogostemon cablin (0.53), Mentha piperita (0.19), Ocimum basilicum (0.09), and Pelargonium gravelens (0.09).

A large variation in the extraction yield of oil from the plants may be due to variation in the plant parts, seasonal variation, and environmental factors for the growth of a particular plant.

Based on microscopic examination of malaria parasite only five plants oils namely Ocimum basilicum, Pogostemon cablin, Papaver somniferum, Ricinus communis and Valeriana jatamansi showed satisfactory results as immersion oil for the examination of malaria parasites.
Performance of different blended mixtures was in order of Mixture 6, Mixture 10, Mixture 4, Mixture 5, Mixture 8, Mixture 7, Mixture 11, Mixture 9, Mixture 1 and Mixture 2 respectively. Results revealed that the mixture 6 consisted of twenty-five percent of each four of Pogostemon cablin, Ocimum basilicum, Papaver somniferum and Ricinus communis $(25: 25: 25: 25)$ showed the best oil to be used for microscopic examination of malaria parasites. Figure 1 and 2 represented microscopic identification of Plasmodium falciparum ring and schizont stages of mixture 6 and synthetic immersion oil respectively.

The physiochemical properties required for an immersion oil including density, viscosity, refractive index and acid value of five oils namely as Ocimum basilicum, Pogostemon cablin, Papaver somniferum, Ricinus communis and Valeriana jatamansi and 11 blended mixtures were are summarized in table 2. Density, refractive index, viscosity and acid values from Ocimum basilicum, Pogostemon cablin, Papaver somniferum, Ricinus communis, Valeriana jatamansi oils and blended mixture 1 , mixture 2, mixture 3 , mixture 4 , mixture 5 , mixture 6 , mixture 7 , mixture 8 , mixture 9 , mixture 10 and mixture 11 ranged $0.916-0.967,1.478-1.501,1.2$ 970 and $0.25-20.0$ respectively. Refractive index and density of the Ocimum basilicum of present study was similar to the reports of Hussain et al., (2008) who investigated the density and refractive index $0.95-0.97 \mathrm{~g} / \mathrm{cm} 3$ and1.4995-1.5045 respectively. Dev et al (2011) reported basil oil refractive index and density as 1.515 and $0.928 \mathrm{~g} / \mathrm{cm} 3$ respectively. Pogostemon cablin oil density, refractive index and acid value were $0.919 \mathrm{~g} / \mathrm{cm} 3,1.499$ and 0.25 . Parganiha (2012) found that the density, refractive index and acid value of the Pogostemon cablin were 0.951-0.991, 1.5089, and1.68-3.93 respectively. Similarly, density, refractive 
index, viscosity and acid value of Papaver somniferum oil were $0.921,1.478,65.4$ and 20.3respectively. Ozcan and Atalay (2006) reported refractive index and acid value of the poppy seed oil as 1.4773 and 1.03.2repectively. Ricinus communis density, refractive index, viscosity and acid value were $0.961,1.481,970$, and 0.3 . respectively which were similar to earlier reports (Deligiannis et al., 2009), Valeriana jatamansi density, refractive index, viscosity and acid value were $0.934,1.491,5.6$, and 0.35 respectively.

Immersion liquids for light microscopy including Cargille immersion oils, currently comply by the ISO/German DIN (2015) which is specified for synthetic immersion oils. As per literature, no specifications have been given for the immersion oil obtained from the plants. Density, refractive index, acid value and viscosity of blended mixture 6 plant oil were $0.945,1.488,33.6$ and 2.36 respectively which are slightly vary from the DIN specification due to the fact that mixture 6 is generated from the plants source. It is to point out that British Pharmacopeia has approved cedar wood plant oil as immersion oil and density, refractive index, viscosity and acid values were similar to the values found for mixture 6 oil.

Stability of oils from Ocimum basilicum, Pogostemon cablin, Papaver somniferum, Ricinus communis, Valeriana jatamansi and mixture 1 , mixture 2, mixture 3 , mixture 4 , mixture 5 , mixture 6 , mixture 7 , mixture 8 , mixture 9 , mixture 10 and mixture 11 with time showed that all were stable under the observation period while non-dryness of the oil extracted from Ocimum basilicum, Pogostemon cablin, Papaver somniferum, Ricinus communis, Valeriana jatamansi and blended mixture 1, mixture 2, mixture 3, mixture 4 , mixture 5 , mixture 6 , mixture 7 , mixture 8 , mixture 9 , mixture 10 , mixture 11were represented very good criteria for the non-drying less than $1 \%$ variation in weight.

GC-MS analysis of patchouli oil revealed presence of alpha.-Copaene $(79.13 \%)$, transCaryophyllene (13.74\%) besides 3, 3, 5, 5, 3', 3', 5', 5'-Octamethyl-DI-(.DELT) and 7Ethylidene-6b 7, 8,8a-tetrahydrocyclobut[a]. Luo et al., (1999) identified pogostone $(30.99 \%)$ in stems, $21.31 \%$ in leaves, patchouli alcohol (10.26\%) in stems, $37.53 \%$ in leaves, deltaguaiene, alpha-guaiene $(2.27 \%)$ in stems, $6.18 \%$ in leaves, seychellene $(1.56 \%)$ in stems, $1.99 \%$ in leaves, alpha-patchoulene, aciphyllene, and trans-caryophyllene $(4.92 \%)$ in stems, $6.75 \%$ in leaves as main constituents from leaves and stem of Pogostemonis (Patchouli) while Cheng et al., (2010) identified patchouli alcohol and pogostone as chemical markers. GC-MS study of Patchouli oil carried out by Micheal (1992) and Daniel (2006), Baby et al., (2007) showed presence of 74 compounds namely Patchouli alcohol, 3-octanone, Benzaldehyde, dimethyl phenol, octanoicacid, Pogostol, 4-methyl-pentanoic-acid,belemene, epiguaipyridine, Ombuine, norpatchoulinol, a-bulnesene, b-patchoulene, epoxycaryophyllen e, p-vinyl-phenol, seychellene, a-bulnesene oxide, b-pinene, Eugenol, pachypodol, nor-patchoulinol, abulnesone, Bulnesol, eugenol, cinnamic aldehyde, patchouli-alcohol, patchouli pyridine, a-guaiene, Cadinene, g-patchoulene, Patchoulipyridine, Methylchavicol, a-guaiene oxide, Camphene, guaiacol, pentanoic-acid, Limonene, a-patchoulene, caryophyllene, guaipyridine, phenol, Pinene, a-pinene, caryophyllene-oxide, heptanoic-acid, pogostol, p-methoxycinnamaldehyde, anethole, cinnamaldehyde, humulene, pogostone, 1,10-epoxy-alphabulnesene, anisaldehyde, cis-2pentylcyclopropylcarboxylic -acid, Limonene, rhamnetin, 1-alpha,5-alphaepoxyalpha-guaiene, 
cycloseychellene, nonanoic-acid, seychellene, 1-beta,5-beta-epoxy-alphaguaiene, apigenin7-o-beta-d-(-6"p-coumaroyl)-glucoside, dpatchoulene, nordehydropatcho ulol,tannin,2methyl-butyric-acid, apigenin-7-o-betaglucoside; benzaldehyde, dehydracetic-acid, norpatchoulenol, trans-2pentylcyclopropylcar boxylic-acid,2-methylhexanoic-acid, azulene, dhelwangin, o-cresol. Bunrathep (2006) identified Sesquiterpenes $\delta$-elemene (t-trace), $\beta$-patchoulene (t), $\beta$-elemene, cis-thujopsene, trans-caryophyllene, $\quad \alpha$-guaiene, $\quad \gamma$ patchoulene, $\alpha$-humulene, $\alpha$-patchoulene, seychellene, valencene, germacrene $D, \beta$ selinene, $\alpha$-selinene, viridiflorene, germacrene A, $\alpha$-bulnesene, 7 -epi- $\alpha$-selinene, oxygenated sesquiterpenes longipinanol, globulol, patchouli alcohol,1-octen-3-ol. Among these, patchouli alcohol $(60.30 \%)$ was the major component, followed by germacrene A (11.73 $\%)$.

Bure and sellier ( 2004) carried out GC/MS study of Indonesian patchouli oil and showed the presence of the following compounds; $\alpha$ pinene, $\delta$-patchoulene, $\beta$-pinene, aciphyllene, limonene, $\delta$-guaiene, $\delta$-elemene, 7 -epi- $\alpha$ selinene, $\alpha$-copaene, norpatchoulenol, $\alpha$ patchoulene, 1,10-epoxy-11-bulnesene, $\beta$ elemene, caryophyllene oxide, cycloseychellene, nortetrapatchoulol, $\beta$ caryophyllene, patchouli alcohol, $\alpha$-guaiene, patchoulenone, seychellene, 9-oxopatchoulol, $\alpha$-humulene, pogostol, $\alpha$-patchoulene, isopatchoulenone, $\quad \gamma$-gurjunene, and germacrene D.

Our study revealed alpha copane and transCaryophyllene as a chemical marker for the presence of patchouli oil. It is to point out that alpha copaene and trans-Caryophyllene has been reported as a major constituent in patchouli oil by Feng and coworkers (1999), Luo et al., (1999) and Silva et al., (2004).

GC-MS analysis of basil oil revealed presence of Linalool L (24.07\%), Estragole (73.47\%),
3,3,5,5,3',3',5',5' Octamethyl -DI-(DELT) (1.55\%),1,1'-bibicyclo (2.2.2) octyl1-4carboxylic acid $(0.51 \%)$, and 1,1'-bibicyclo (2.2.2) octyl1-4-carboxylic acid (0.4\%). Kathirvel and Ravi (2012) reported chemical compositions of basil fresh leaves identified by GC-MS: 11 components were identified. The major constituents were found to be methyl cinnamate $(70.1 \%)$, linalool $(17.5 \%), \quad \beta$-elemene $(2.6 \%)$ and camphor $(1.52 \%)$. Linalool was the main constituent of O. basilicum essential oil (56.7-60.6\%), followed by epi-a-cadinol (8.6-11.4\%), abergamotene (7.4-9.2\%), c-cadinene (3.3$5.4 \%)$, germacrene D (1.1-3.3\%) and camphor (1.1-3.1\%) (Hussain et al., 2008).

Present study revealed that Linalool L and Estragole as chemical marker for the identification of basil oil. Estragol, linalool, methyl eugenol, geraniol, methyl cinnamate, bergamotene, $\alpha$-cubebene, germacrene $D, \beta$ elemene, 1,8-cineole, methyl cinnamate, $\alpha$ cadinol and limonene are considered as the main constituents and chemotypes of basil from different parts of the world (Koba et al., 2009, Zhang and coworkers (2009), Abduelrahman et al., (2009) and Vani and coworkers (2009). Jirovetz and Buchbauer (2001) found a high level of linalool (71.4\%) in $O$. basilicum essential oil from Bulgaria. According to Gurbuz et al., (2006), linalool $(41.2 \%)$ was the main compound, identified in the hydro-distilled $O$. basilicum essential oil from Turkey. Hassanpouraghdam et al., (2010) reported Linalool in leaves as major component in Basil oil.

GC-MS analysis of poppy seed revealed presence of hexadecanoic acid ethyl ester (1.40), 9-octadecanoic acid (3.55), 9,12,15octadecatrienoic acid, (2-phenyl-1,3dioxolan-4-YL) methyl E (0.22), hexadecanoic acid phenyl methyl ester (30.73), ethyl(9Z,12Z)-9,12-Octadecadienoate (11.23),9,12-Octadecadienoic acid (Z, Z) (41.59), and Heneicosyl pentafluoro- 
propionate (7.82). Ozcan and Atalay (2006) reported linoleic acid high in all the samples, and varied between 52.6 to $71.50 \%$ while Rahimi et al., (2011) studied opium poppy seed oils from Turkey where the main fatty acids were linoleic (56.4- 69.2\%), oleic (16.1$19.4 \%$ ) and palmitic acids (10.6- 16.3\%). Singh et al., (1990) stated poppy seeds contained up to $50 \%$ oil and Indian cultivars have high levels of oleic and linoleic acids. Similarly, some researchers have reported that the linoleic (C18:2), oleic (C18:1) and palmitic acids (C16:0) are major fatty acids in the poppy seed oil (Erinc et al., 2009, Singh et al., (1990), Sener et al., (1999), Bezakova et al., (1994), Bajpai (1999), Bozan and Temelli (2008) and Luthra and Singh, 1989).

Table.1 Scientific name, family and common names, raw plant parts used for extraction and method of extraction the plants under investigation

\begin{tabular}{|c|c|c|c|c|c|}
\hline S.no. & Scientific name & Family & $\begin{array}{l}\text { Common } \\
\text { name }\end{array}$ & $\begin{array}{c}\text { Raw material } \\
\text { used for oil } \\
\text { extraction }\end{array}$ & $\begin{array}{l}\text { Method of } \\
\text { extraction }\end{array}$ \\
\hline 1. & $\begin{array}{l}\text { Cymbopogon } \\
\text { martini }\end{array}$ & $\begin{array}{l}\text { Graminae } \\
\text { (Poaceae) }\end{array}$ & Palmarosa & Leaves & $\begin{array}{l}\text { Steam } \\
\text { Distillation }\end{array}$ \\
\hline 2. & $\begin{array}{l}\text { Pogostemon } \\
\text { cablin }\end{array}$ & $\begin{array}{l}\text { Lamiaceae } \\
\text { (Labiatae) }\end{array}$ & Patchouli & Leaves & $\begin{array}{l}\text { Steam } \\
\text { Distillation }\end{array}$ \\
\hline 3. & $\begin{array}{l}\text { Melaleuca } \\
\text { alternifolia }\end{array}$ & Myrtaceae & Tea Tree & Leaves & $\begin{array}{l}\text { Steam } \\
\text { Distillation }\end{array}$ \\
\hline 4. & $\begin{array}{l}\text { Cedrus } \\
\text { deodara }\end{array}$ & Pinaceae & Cedar wood & Cone & $\begin{array}{l}\text { Steam } \\
\text { Distillation }\end{array}$ \\
\hline 5. & $\begin{array}{l}\text { Pelargonium } \\
\text { gravelens }\end{array}$ & Geraniaceae & Geranium & Leaves & $\begin{array}{l}\text { Steam } \\
\text { Distillation }\end{array}$ \\
\hline 6. & $\begin{array}{l}\text { Cymbopogon } \\
\text { nardus }\end{array}$ & $\begin{array}{l}\text { Graminae } \\
\text { (Poaceae) }\end{array}$ & Citronella & Leaves & $\begin{array}{l}\text { Steam } \\
\text { Distillation }\end{array}$ \\
\hline 7. & $\begin{array}{l}\text { Pinus } \\
\text { roxberghii }\end{array}$ & Pinaceae & Pine & Needle & $\begin{array}{l}\text { Steam } \\
\text { Distillation }\end{array}$ \\
\hline 8. & $\begin{array}{l}\text { Ocimum } \\
\text { basilicum }\end{array}$ & $\begin{array}{l}\text { Lamiaceae } \\
\text { (Labiatae) }\end{array}$ & Basil & Leaves & $\begin{array}{l}\text { Steam } \\
\text { Distillation }\end{array}$ \\
\hline 9. & $\begin{array}{l}\text { Mentha } \\
\text { piperita }\end{array}$ & $\begin{array}{l}\text { Lamiaceae } \\
\text { (Labiatae) }\end{array}$ & Peppermint & Leaves & $\begin{array}{l}\text { Steam } \\
\text { Distillation }\end{array}$ \\
\hline 10. & $\begin{array}{l}\text { Cymbopogon } \\
\text { citratus }\end{array}$ & $\begin{array}{l}\text { Graminae } \\
\text { (Poaceae) }\end{array}$ & Lemongrass & Leaves & $\begin{array}{l}\text { Steam } \\
\text { Distillation }\end{array}$ \\
\hline 11. & $\begin{array}{l}\text { Eucalyptus } \\
\text { citriodora }\end{array}$ & Myrtaceae & Lemon gum & Leaves & $\begin{array}{l}\text { Steam } \\
\text { Distillation }\end{array}$ \\
\hline 12. & Jatropa curcas & Euphorbiaceae & $\begin{array}{l}\text { Ratanjyot, } \\
\text { Physic Nut }\end{array}$ & Seed & $\begin{array}{l}\text { Solvent } \\
\text { Extraction }\end{array}$ \\
\hline 13. & $\begin{array}{l}\text { Pongammia } \\
\text { pinnata }\end{array}$ & $\begin{array}{l}\text { Leguminosae, } \\
\text { (Fabaceae) }\end{array}$ & Karanja oil & Seed & $\begin{array}{l}\text { Solvent } \\
\text { Extraction }\end{array}$ \\
\hline 14. & $\begin{array}{l}\text { Terminelia } \\
\text { chebulla }\end{array}$ & Combretaceae & Harra & Seed & $\begin{array}{l}\text { Solvent } \\
\text { Extraction }\end{array}$ \\
\hline 15. & Terminellia & Combretaceace. & Bahera, & Seed & Solvent \\
\hline
\end{tabular}




\begin{tabular}{|c|c|c|c|c|c|}
\hline & belerica & & $\begin{array}{l}\text { bastard } \\
\text { myrobalan }\end{array}$ & & Extraction \\
\hline 16. & $\begin{array}{l}\text { Sapindus } \\
\text { mukrossi }\end{array}$ & Sapindaceae & $\begin{array}{l}\text { Reetha, } \\
\text { soapnut }\end{array}$ & Seed & $\begin{array}{l}\text { Solvent } \\
\text { Extraction }\end{array}$ \\
\hline 17. & $\begin{array}{l}\text { Mimusops } \\
\text { elengi }\end{array}$ & Sapotaceae & Maulsari & Seed & $\begin{array}{l}\text { Solvent } \\
\text { Extraction }\end{array}$ \\
\hline 18. & $\begin{array}{l}\text { Bauhinia } \\
\text { retusa }\end{array}$ & Caesalpiniaceae & Devanagari & Seed & $\begin{array}{l}\text { Solvent } \\
\text { Extraction }\end{array}$ \\
\hline 19. & $\begin{array}{l}\text { Bauhinia } \\
\text { purpurea }\end{array}$ & Leguminosae & $\begin{array}{l}\text { Orchid tree, } \\
\text { purple } \\
\text { bauhinia }\end{array}$ & Seed & $\begin{array}{l}\text { Solvent } \\
\text { Extraction }\end{array}$ \\
\hline 20. & $\begin{array}{l}\text { Pithecolobium } \\
\text { dulci }\end{array}$ & Fabaceae & $\begin{array}{l}\text { Madras thorn, } \\
\text { jungle jalebi }\end{array}$ & Seed & $\begin{array}{l}\text { Solvent } \\
\text { Extraction }\end{array}$ \\
\hline 21. & $\begin{array}{l}\text { Ceiba } \\
\text { pentandra }\end{array}$ & Malvaceae & Kapok & Seed & $\begin{array}{l}\text { Solvent } \\
\text { Extraction }\end{array}$ \\
\hline 22. & $\begin{array}{l}\text { Moringa } \\
\text { oleferia }\end{array}$ & Moringaceae & Senjana & Seed & $\begin{array}{l}\text { Solvent } \\
\text { Extraction }\end{array}$ \\
\hline 23. & Sterculia foetida & Malvaceae & Wild almond & Seed & $\begin{array}{l}\text { Solvent } \\
\text { Extraction }\end{array}$ \\
\hline 24. & Junglans regia & Juglandaceae. & Walnut & Seed & $\begin{array}{l}\text { Solvent } \\
\text { Extraction }\end{array}$ \\
\hline 25. & $\begin{array}{l}\text { Michalia } \\
\text { champaca }\end{array}$ & Magnolia & Champaca & Seed & $\begin{array}{l}\text { Solvent } \\
\text { Extraction }\end{array}$ \\
\hline 26. & $\begin{array}{l}\text { Cesalpinia } \\
\text { boundecella }\end{array}$ & Caesalpiniaceae & $\begin{array}{l}\text { Gray Nicker, } \\
\text { Kantkarej }\end{array}$ & Seed & $\begin{array}{l}\text { Solvent } \\
\text { Extraction }\end{array}$ \\
\hline 27. & Aleurites fordii & Euphorbiaceae & Tung & Seed & $\begin{array}{l}\text { Solvent } \\
\text { Extraction }\end{array}$ \\
\hline 28. & Olea europaea & Oleaceae & Olive & Seed & $\begin{array}{l}\text { Solvent } \\
\text { Extraction }\end{array}$ \\
\hline 29. & $\begin{array}{l}\text { Papaver } \\
\text { somniferum }\end{array}$ & Papaveraceae & Opium poppy & Seed & $\begin{array}{l}\text { Solvent } \\
\text { Extraction }\end{array}$ \\
\hline 30. & $\begin{array}{l}\text { Ricinus } \\
\text { Communis }\end{array}$ & Euphorbiaceae & Castor & Seed & $\begin{array}{l}\text { Solvent } \\
\text { Extraction }\end{array}$ \\
\hline 31. & $\begin{array}{l}\text { Valeriana } \\
\text { jatamansi }\end{array}$ & Caprifoliaceae & Jatamansi & Root & $\begin{array}{l}\text { Solvent } \\
\text { Extraction }\end{array}$ \\
\hline 32. & $\begin{array}{l}\text { Psoralea } \\
\text { corylifolia }\end{array}$ & Fabaceae & Babchi & Seed & $\begin{array}{l}\text { Solvent } \\
\text { Extraction }\end{array}$ \\
\hline
\end{tabular}


Table.2 Physicochemical properties of plant oils and blended mixtures used as immersion oil for microscopic examination of malaria parasites

\begin{tabular}{|l|l|c|c|c|c|}
\hline $\begin{array}{l}\text { S. } \\
\text { No }\end{array}$ & \multicolumn{1}{|c|}{$\begin{array}{c}\text { Name of the oils/ } \\
\text { Mixtures }\end{array}$} & $\begin{array}{c}\text { Density } \\
\text { (g/cm3) }\end{array}$ & $\begin{array}{c}\text { Refractive } \\
\text { Index (RI) }\end{array}$ & $\begin{array}{c}\text { Viscosity at } \\
\text { 5 RPM(cP) }\end{array}$ & $\begin{array}{c}\text { Acid Value } \\
\text { (mg/KOH) }\end{array}$ \\
\hline 1 & $\begin{array}{l}\text { Ocimum basilicum } \\
\text { (Basil) }\end{array}$ & 0.937 & 1.501 & 1.2 & 0.57 \\
2 & $\begin{array}{l}\text { Pogostemon cablin } \\
\text { (Patchouli ) }\end{array}$ & 0.919 & 1.499 & 9 & 0.25 \\
3 & Papaver somniferum & 0.921 & 1.478 & 65.4 & 3.49 \\
4 & (Poppy seed) & 0.961 & 1.481 & 970 & 0.3 \\
& Ricinus communis & & & & \\
5 & (Castor) & 0.934 & 1.491 & 5.6 & 0.49 \\
6 & Valeriana jatamansi & & & & \\
7 & Matamansi) & 0.927 & 1.492 & 13.8 & 3.76 \\
8 & Mixture1 & 0.916 & 1.488 & 29 & 3.36 \\
9 & Mixture3 & 0.937 & 1.491 & 19.4 & 2.76 \\
10 & Mixture4 & 0.947 & 1.494 & 31.2 & 0.48 \\
11 & Mixture5 & 0.936 & 1.491 & 102 & 0.31 \\
12 & Mixture7 & $\mathbf{0 . 9 4 5}$ & $\mathbf{1 . 4 8 8}$ & $\mathbf{3 3 . 6}$ & $\mathbf{1 . 7 0}$ \\
13 & Mixture8 & 0.967 & 1.478 & 206 & 3.15 \\
14 & Mixture9 & 0.922 & 1.485 & 18.6 & 0.29 \\
15 & Mixture10 & 0.932 & 1.485 & 16.8 & 0.29 \\
16 & Mixture11 & 0.935 & 1.478 & 16 & 0.5 \\
& & 0.919 & 1.478 & 24.4 & 1.45 \\
\hline
\end{tabular}

Table.3 Result of In-house validation

\begin{tabular}{|c|c|c|c|c|}
\hline S. No. & Examiner Code & $\begin{array}{c}\text { Total slides } \\
\text { examined }\end{array}$ & \multicolumn{2}{|c|}{ Observations } \\
\cline { 3 - 5 } & & & $\begin{array}{c}\text { Very Good ++++ } \\
(\%)\end{array}$ & $\begin{array}{c}\text { Good } \\
+++ \\
(\%)\end{array}$ \\
\hline 1 & & & & $4(20)$ \\
2 & NC & 20 & $16(80)$ & $5(25)$ \\
3 & MR & 20 & $15(75)$ & $4(20)$ \\
4 & AR & 20 & $16(80)$ & $3(15)$ \\
\hline
\end{tabular}


Fig.1a\&b Microscopic identification of Plasmodium falciparum ring stage using synthetic immersion oil and microscopic identification of Plasmodium falciparum ring stage using Mixture 6 immersion oil

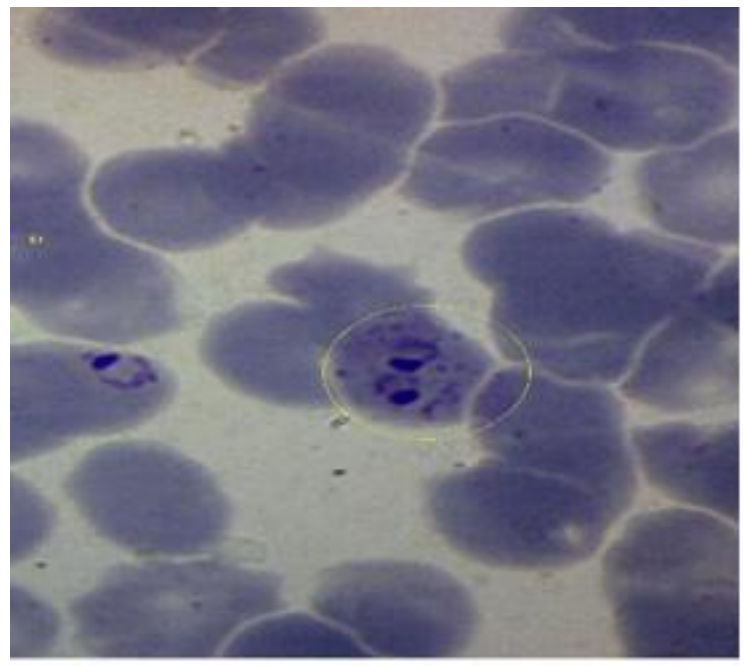

Figure 1a

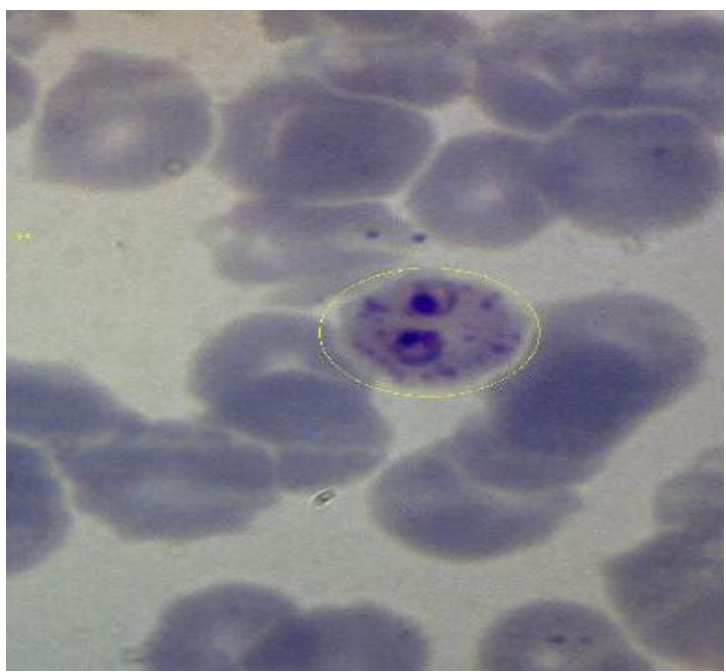

Figure $1 b$

Fig.2a\&b Microscopic identification of Plasmodium falciparum schizont stage using synthetic immersion oil and Microscopic identification of Plasmodium falciparum schizont stage using Mixture 6 immersion oil

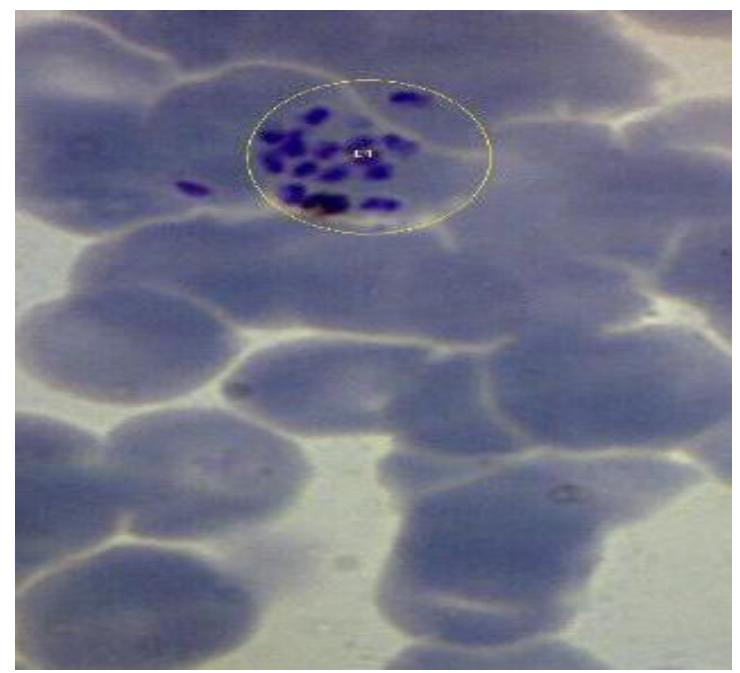

Figure 2a

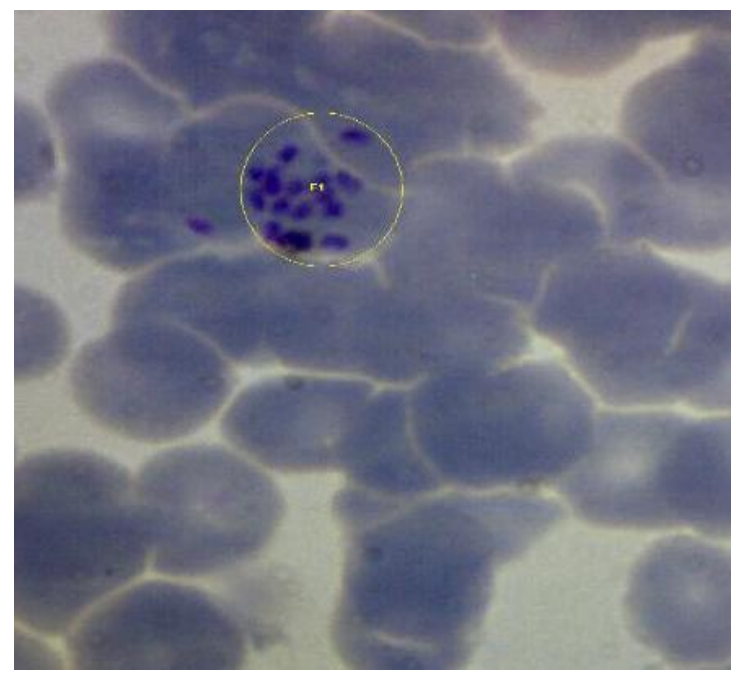

Figure $2 b$ 
Table.4 Result of external validation

\begin{tabular}{|c|c|c|c|c|}
\hline S. No. & Examiner Code & $\begin{array}{c}\text { Total slide } \\
\text { examine }\end{array}$ & \multicolumn{2}{|c|}{ Observations } \\
\cline { 4 - 5 } & & & $\begin{array}{c}|c| \\
\text { Very Good ++++ } \\
\text { (\%) }\end{array}$ & $\begin{array}{c}\text { Good +++ } \\
\text { (\%) }\end{array}$ \\
\hline 1 & HIM & 20 & $17(85)$ & $3(15)$ \\
2 & SG & 20 & $16(80)$ & $4(20)$ \\
3 & MET & 20 & $18(90)$ & $2(10)$ \\
4 & GH & 20 & $17(85)$ & $3(15)$ \\
\hline
\end{tabular}

HIM: Himalayan Hospital Jolly Grant, Dehradun, SG: Shri Guru Ram Rai Hospital Dehradun, MET: Metro Hospital SIDCUL, Hardwar and GH: Government Hospital Hardwar.

GC-MS analysis of mixture 6 clearly identified presence of alpha copane and transCaryophyllene as a chemical marker for the presence of patchouli oil, Linalool L and Estragole as chemical marker for basil oil and phenyl methyl ester, and 9,12Octadecadienoic acid for the presence of poppy seed oil besides other minor components peaks as stated in results. It is to note that no peak was detected for castor oil due to lack of sample character and detection procedure.

Summary of in-house validation of blended mixture 6 carried out by four microscopists is given in table 3 . It is to point out that all four microscopists have classified mixture 6 as very good immersion oil (range 67-81\%) and good (19-33\%) for the examination of malaria parasites while the results of external validation by four different hospitals/ laboratory are given in table 4. Certifications by four Head/Incharge of the hospitals clearly revealed that the developed plant oil mixture 6 possessed very good property as immersion oil for the examination of malaria parasites and may develop as an alternative of synthetic immersion oil.

Present study was aimed to find out plant oil based immersion oil as an alternative to synthetic immersion oil which showed airborne contact dermatitis, burning pruritus and urticarial-like lesions on the face and forearms and carcinogenic, a hazard to the human environment. A blended mixture consisted of twenty-five percent of Pogostemon cablin, Ocimum basilicum, Papaver somniferum and Ricinus communis (25:25:25:25) showed very good results for microscopic examination of malaria parasites.

\section{Acknowledgements}

Financial support from Uttarakhand Council of Science and Technology (UCOST), Dehradun is sincerely acknowledged.

\section{References}

Abduelrahman, A.H.N., Elhussein, S.A., Osman, N.A.I. and Nour, A.H. 2009. Morphological Variability and Chemical Composition of Essential Oils from Nineteen Varieties of Basil (Ocimum basilicum L.) Growing in Sudan. Int. J. Chem. Technol., 1: 1-10.

Baby, P., Skaria, Joy, P.P., Samuel Mathew, Qracy. 2007. Aromatic Plants: Horticulture Science Series, Laxmi Art Creations New Delhi.

Bajpai, S., Prajapati, S., Luthra, R., Sharma, S., Naqvi, A. and Kumar, S. 1999. Variation in the seed and oil yields and oil quality in the Indian germplasm of opium poppy Papaver somniferum. 
Genetic Res. Crop Evol., 46(5): 435439.

Barkatullah, I.M., Abdur, R. and Rahman, I.Ur. 2012. Physicochemical Characterization of Essential and Fixed Oils of Skimmia laureola and Zanthoxylum armatum. Middle-East J. Med. Plants Res., 1(3): 51-58.

Bozan Berrin, Temelli Feral. 2008. Chemical composition and oxidative stability of flax, safflower and poppy seed and seed oils. Biores. Technol., Volume 99, Issue 14, Pages 6354-6359.

British Pharmacopeia. 1963. The pharmaceutical press, 17 Bloomsbury Square London WC1, UK, pp 144.

Bure, C.M., Sellier, N.M. 2004. Analysis of the essential oil of Indonesian Patchouli (Pogostemon cablin Benth) using GC/MS (EI/CI). J. Essent. Oil Res., 16: $17-1$.

Cheng, X., Zhang, H., Yang, L., Lin, Z., Zou, Z., Yu, C. 2010. Identification method with significant specificity of volatile oil of Pogostemon cablin. Zhongguo Zhong Yao Za Zhi, 35(17): 2270-2272.

Daniel, M. 2006. Medicinal Plants: Chemistry and Properties. Science Publishers, Enfield, New Hampshire, United states, 250pp.

Deligiannis, A., Anastopoulos, G., Karavalakis, G. and Lois, E. 2009. Castor (Ricinus communis L) seed oil as an alternative feedstock for the production of biodiesel proceeding of the 11thConference on Environmental Science and Technology, at Chania, Crete, Greece.

Dev, N., Das, A.K., Hossain, M.A. and Rahman, S.M.M. 2011. Chemical Compositions of Different Extracts of Ocimum basilicum Leaves. J. Sci. Res., 3(1): 197-206.

Dua, V.K., Kumar, A., Pandey, A.C., and Kumar, S. 2013. Insecticidal and genotoxic activity of Psoralea corylifolia Linn. (Fabaceae) against Culex quinquefasciatus Say, 1823. Parasite and Vectors, 4: 6-13.

FAO Corporate Document Repository. 1995. Flavors and fragrances of plant origin; cedar wood oil, Chapter 10, Pages 111.

Feng, Y., Guo, F., Luo, J. 1999. GC-MS analysis of volatile oil of Herba pogostemonis collected from Leizhou country. Zhong Yao Cai, 22: 241-243.

Fujioka, T., Fukunaga, Y., Mine, T. 2009. Microscope immersion oil. Patent no. EP2048193 A1

Hassanpouraghdam, M.B., Gohari, G.R., Tabatabaei, S.J., Dadpour, M.R. 2010. Inflorescence and leaves essential oil composition of hydroponically grown Ocimum basilicum L. J. Serbe Chem. Soc., 75: 1361-8.

Hussain, A.I., Anwar, F., Sherazi, S., Tufail, H., Przybyiski, R. 2008. Chemical composition, antioxidant, antimicrobial activities of basil (Ocimum basilicum) essential oils depend on seasonal variations. Food Chem., Volume 108, Issue 3, 1 June, Pages 986-995.

Kathirvel, P., Ravi, S. 2012. Chemical composition of the essential oil from basil (Ocimum basilicum Linn.) and its in vitro cytotoxicity against $\mathrm{HeLa}$ and HEp-2 human cancer cell lines and NIH 3T3 mouse embryonic fibroblasts. Nat. Prod. Res., 26(12): 1112-8.

Koffi, K., Poutouli, P.W., Raynaud Christine, Chaumont Jean-Pierre and Sanda Komla 2009. Chemical composition and antimicrobial properties of different basil essential oils chemotypes from Togo. Bangl. J. Pharmacol., 4: 1-8.

Liva, M. 1986. Immersion oil system. United States Patent No. 4587042.

Luo, J., Feng, Y., Guo, X., Li, X. 1999. GCMS analysis of volatile oil of Herba pogostemonis. collected from Gaoyao country. Zhong Yao Cai, 22: 25-28.

Micheal Tierra. 1992. Planetary Herbol., 
Lotus Press Twin Lakes, 251.

Nzikou, J.M., M. Mvoula-Tsieri, L. Matos, E. Matouba, A.C Ngakegni, M. Linder and S. Desobry. 2007. Solanum nigrum L. seeds as an alternative source of edible lipids and nutriment in Congo Brazzaville. J. Appl. Sci., 7: 1107-1115.

Ozcan musa and chalchat jean-clause. 2002. Essential Oil Composition of Ocimum basilicum L. and Ocimum minimum L. in Turkey Czech. J. Food Sci., Vol. 20, No. 6: 223-228.

Parganiha Deepak. 2012. Process Technology for Extraction of Essential Oil from Patchouli (Pogostemon cablin Benth.). Indira Gandhi Krishi Vishwa Vidhyalaya, Raipur Roll No: 13938 ID No. 310106004
Silva, M.A.S., Ehlert, P.A.D., Ming, L.C., Marques, M.O.M. 2004. Composition and chemical variation during daytime of constituents of the essential oil of Pogostemon patchouli Pellet leaves. Acta Hortic., 629: 145-147.

Vani, S.R., Cheng, S.F., Chuah, C.H. 2009. Comparative Study of Volatile Compounds from Genus Ocimum. Am. J. Appl. Sci., 6(3): 523-528.

Zhang, J.W., Li, S.K., Wu, W.J. 2009. The Main Chemical Composition and in vitro Antifungal Activity of the Essential Oils of Ocimum basilicum Linn. var. pilosum (Willd.) Benth Mol.,14(1): 273-278; doi:10.3390/molecules14010273

\section{How to cite this article:}

Ayushi Rastogi, V.K. Dua, V.K. Varshney, N.C. Gupta and Sumit Kumar. 2017. Herbal Immersion Oil for Microscopic Identification of Malaria Parasites. Int.J.Curr.Microbiol.App.Sci. 6(8): 2267-2279. doi: https://doi.org/10.20546/ijcmas.2017.608.266 